\title{
Gap theorems for minimal submanifolds in $R^{n+1}$
}

\author{
LEI NI ${ }^{1}$
}

\section{Introduction.}

Let $M^{m}$ be a compact immersed minimal submanifold of dimension $m$ in the unit $S^{n}(1)$. It was proved by J. Simons in [S] that if $\|A\|^{2}<\frac{m(n-m)}{2 n-2 m-1}$, where $A$ is the second fundamental form, then $M$ is totally geodesic. It was also proved by S.S. Chern, M. Do Carrio and S. Kobayashi using the moving franie in [C.D-K] later. For the minimal submanifolds in $R^{n+1}$ it was proved in [Al] tl:at there is a similar theorem for the volume growth. More precisely, Allard showed that if $M^{m}$, a minimal submanifold of $R^{n+1}$ has Euclidear: volume growth and the density function $\Theta_{M}(x, r)=\frac{\operatorname{Vol}\left(M \cap B_{r}(x)\right)}{V_{0}^{m}(1) r^{m}}<1+\delta$, for some surall positive number $\delta$, inen $M$ is totally geodesic. On the other hand, it was shown in [Ar]], [F-C] and [Ty] that when $M^{m}$ is a minimal submanifold (of dimension $m$ ) in $R^{n+1}$, the total scalar curvature $\int_{M}\|A\|^{m} d v$ is closed related to the topology and the Morse index of $M$. More recently it was shown in [S-Z] that if $M$ is a stable minimal hypersurface with finite total scalar curvature ther. $M$ is totally geodesic. In this short note we will show tlsal threre are some gap theorems for the total scalar curvature. These may be thought as the analogy of the above mentioned Simons' theorem for the minimal subrinanifolds in $R^{n+1}$. More precisely we can show the following result: (We should point out that we do not need the stability assumption, wriacis is essential in the above mentioned Shen-Zhu's result in [S-Z] )

Theorem A. Let $M^{n}(\{\geq 4)$ be a complete minimal immersed hypersurface. in $R^{n+}$ Then there exists a constint $C_{1}(n)=\sqrt{\frac{4(n-1)}{(n-2) n} S^{-1}(n)}>0$ acul that if

$$
\left(\int_{M}\|A\|^{n} d v\right)^{\frac{1}{n}}<C_{1}(n)
$$

\footnotetext{
1 The research was partially supporied by NSF grant DMS-0196405.
} 
$M$ must be totally geodesic. Here $A$ is the second fundamental form of $M$ and $S(n)$ is the constant in the $L^{2}$-Sobolev inequality. (For example, using the $L^{1}$-Sobolev constant provided in $[\mathrm{M}-\mathrm{S}]$, one can show that $S(n)=$ $\left(\frac{4^{n+1}(2 \dot{n}-2)}{\omega_{n}^{1 / n}(n-2)}\right)^{2}$ will be big enough, where $\omega_{n}$ is the volume of the unit ball in $R^{n}$.)

In the proof of Theorem A we need first to show the following result about the ends of minimal submanifolds, which can be viewed as a gap theorem for the number of ends.

Theorem B. Let $M^{m}(m \geq 3)$ be a complete minimal immersed submanifold of dimension $m$ in $R^{n+1}$. Then there exists a constant $C_{2}(m)=$ $\sqrt{\frac{m}{m-1} S^{-1}(m)}>0$ such that if

$$
\left(\int_{M}\|A\|^{m} d v\right)^{\frac{1}{m}}<C_{2}(m)
$$

$M$ has only one end. Here, as in Theorem $A, A$ is the second fundamental form and $S(m)$ is the constant in the $L^{2}$-Sobolev inequality.

The above gap theorem further demonstrates the fact that there is a close relation between the topology of the minimal submanifold $M^{m}$ and the total scalar curvature $\int_{M}\|A\|^{m} d v$.

Concerning our second result, there are a few previous results which we should mention. First it was proved in $[\mathrm{F}-\mathrm{C}]$, for the minimal surface in $R^{3}$, that the finiteness of the total scalar curvature implies finiteness of the Morse index as well as finite many ends. Later this statement was generalized to the high dimension minimal hypersurfaces in [Ty]. More recently, using the function theory, [C-S-Z] proved that if $M$ is a stable minimal hypersurface in $R^{n+1}$ then $M$ has only one end. Our Theorem $\mathrm{B}$ and related results in the next section conclude that $M$ has only one end or finite many ends without assuming that $M$ is stable or of codimension one. Very recently, [L-W] proved the Liouville property for minimal submanifold $M$, whose density function $\Theta_{M}(x, r)$ satisfies that $\Theta_{M}(x, r) \leq \bar{\Theta}<2$. Namely they showed that there is no bounded harmonic functions on such minimal submanifolds. Combining with the observations in the next section of this paper, as a corollary, we know that $M$ has at most one end if its density function satisfies $\Theta_{M}(x, r) \leq \bar{\Theta}<2$. Therefore one can view their result as a gap type theorem for the number of ends. The constant $C_{1}$ and $C_{2}$ in our theorems are far away from the sharpest because of the dependency on 
the $L^{2}$-Sobolev constant on minimal submanifolds. It would be very nice if one can find the best constant as Simons' theorem in $[\mathrm{S}]$ and classify all the minimal hypersurfaces for which the equality holds as in [C-D-K]. Finally we should mention that the argument we used can also sharpen a lower bound estimate for the first eigenvalue of a Schrödinger operator of Li-Yau [L-Y2], therefore sharpens the upper bound for the number of bound states obtained in [L-Y2] (cf. remarks before Theorem 3.2).

Acknowledgement. The author would like to thank Professor Peter Li for helpful suggestions.

\section{Preliminaries.}

We first establish some basic results on the harmonic function theory on minimal submanifolds of $R^{n+1}$. Using the bounded harmonic functions with finite Dirichlet integral we then show a couple of results concerning the finiteness of the number of ends for the minimal submanifolds in $R^{n+1}$.

Let $M^{m}$ be a minimal submanifold of dimension $m \geq 3$ in $R^{n+1}, p \in M$ be a point on $M$. Let $A$ be the second fundamental form of $M$ and $\|A\|$ be the length of the second fundamental form. Let $r_{p}(x)$ be the extrinsic distance function of $R^{n+1}$ with respect to $p$ and $\rho_{p}(x)$ be the intrinsic distance function. We shall also use the following conventions:

$$
\begin{aligned}
& \tilde{B}_{p}(a):=\text { the ball of radius } a \text { centered at } p \text { in } R^{n+1} \\
& D_{p}(a):=\tilde{B}_{p}(a) \cap M \\
& B_{p}(a):=\text { the geodesic ball of radius } a \text { centered at } p \text { in } M .
\end{aligned}
$$

The following lemma will be very convenient to use in the construction of bounded harmonic functions on $M$.

Lemma 2.1. Let $M^{m}(m \geq 3)$ be a m-dimensional minimal submanifold in $R^{n+1}$. Then there exist minimal positive Green's function $G(x, y)$ on $M$ such that $\lim _{r_{y}(x) \rightarrow \infty} G(x, y)=0$.

Proof. Let $D_{p}(a)$ be as the above. The heat-kernel comparison of Cheng-LiYau implies that

$$
H_{D_{p}(a)}(x, y, t) \leq \bar{H}_{a}\left(r_{y}(x), t\right),
$$

where $\bar{H}_{a}\left(r_{y}(x), t\right)$ is the Dirichlet heat-kernel of $\tilde{B}^{m}(a)$ in $R^{m}$ (the ball of 
radius $a$ centered at origin). This implies that

$$
H_{D_{p}(a)}(x, y, t) \leq \frac{1}{(4 \pi t)^{\frac{n}{2}}} \exp \left(-\frac{\left|r_{y}(x)\right|^{2}}{4 t}\right) .
$$

Taking $a \rightarrow \infty$ we have that

$$
H(x, y, t) \leq \frac{1}{(4 \pi t)^{\frac{n}{2}}} \exp \left(-\frac{\left|r_{y}(x)\right|^{2}}{4 t}\right) .
$$

Integrating along the time direction, we have that

$$
G(x, y) \leq \frac{1}{m(m-2) \omega_{m}}\left|r_{y}(x)\right|^{2-m} .
$$

Remark. One can also construct Green's function directly as in [L-T1] by compact exhaustion and applying the Sobolev inequality to prove that the limit exists. By using the heat kernel estimate we can get the upper bound of the Green's function as a consequence.

As a corollary of the heat kernel estimate we have the following:

Corollary 2.2 (Mean-Value inequality). (See [C-L-Y], [M-S].) Let $M^{m}$ be a minimal submanifold in $R^{n+1}$. Suppose $f$ is a nonnegative subharmonic function defined on $M^{m}$. Then

$$
\begin{gathered}
f(p) \leq \frac{1}{m \omega_{m} a^{m-1}} \int_{\partial D_{p}(a)} f(x) d A \\
f(p) \leq \frac{1}{\omega_{m} a^{m}} \int_{D_{p}(a)} f(x) d v
\end{gathered}
$$

where $\omega_{m}$ is the volume of the unit ball in $R^{m}$.

Proof. The first part proof can be found in [C-L-Y] or [M-S]. For the second part we only need to apply the co-area formula and note that $|\nabla r| \leq 1$. More precisely

$$
\begin{aligned}
f(p) \omega_{m} a^{m} & =\int_{0}^{a} f(p) m \omega_{m} s^{m-1} d s \leq \int_{0}^{a} \int_{\partial D_{p}(s)} f(x) d A d s \\
& \leq \int_{0}^{a} \int_{\partial D_{p}(s)} \frac{f(x)}{|\nabla r|} d A d s=\int_{D_{p}(a)} f(x) d v .
\end{aligned}
$$

Applying an argument of Varopoulos (cf. [V]), one can have Sobolev inequality as a corollary of the heat-kernel estimate of Cheng-Li-Yau. 
Corollary 2.3 (Sobolev Inequality). (See also [M-S].) Let $M^{m}$ be a minimal submanifold in $R^{n+1}$, then there exists constant $S=S(m)$ such that

$$
\left(\int_{M} \phi^{\frac{2 m}{m-2}} d v\right)^{\frac{m-2}{m}} \leq S(m) \int_{M}|\nabla \phi|^{2} d v
$$

for any compact supported smooth function $\phi$ on $M$.

Remark. In fact, Michael and Simon proved a stronger version of Sobolev inequality, so-called $L^{1}$-Sobolev inequality, as follows:

$$
\left(\int_{M} \phi^{\frac{m}{m-1}} d v\right)^{\frac{m-1}{m}} \leq S_{1} \int_{M}|\nabla \phi| d v
$$

In fact, they showed that $S_{1}(m)=\frac{4^{m+1}}{\omega_{m}^{1 / m}}$ will be enough to have the above inequality. Using this fact, we can have a lower bound for $S(m)$ in the inequality (2.3). In fact $S(m)=\left(\frac{4^{m+1}(2 m-2)}{\omega_{m}^{1 / m}(m-2)}\right)^{2}$ will be big enough to have inequality (2.3).

Once we have the minimal positive Green's function on $M$ we can apply the scheme of [L-T2] to construct barrier functions at each end of $M$.

Lemma 2.4. Let $M^{m}$ be a minimal submanifold in $R^{n+1}, K \subset M$ be a compact subset in $M$, and let $E_{i}$ be the ends with respect to $K$, then there exist harmonic functions $g_{i}$ on $E_{i}$ which satisfy that

$$
\left.g_{i}(x)\right|_{\partial E_{i}}=1, \lim _{x \rightarrow \infty} g_{i}(x)=0, \int_{E_{i}}\left|\nabla g_{i}\right|^{2} d v<\infty .
$$

Proof. This result is essentially proved in [L-T2]. For the sake of completeness we sketch the proof here. Let $g_{i}^{j}(x)$ be harmonic function defined on $E_{i} \cap D_{p}\left(r_{j}\right)$ and satisfies $\left.g_{i}^{j}(x)\right|_{\partial E_{i}}=1,\left.g_{i}^{j}(x)\right|_{\partial D_{p}\left(r_{j}\right) \cap E_{i}}=0$. We have the estimate $g_{i}^{j}(x) \leq C G(p, x)$, for some constant $C$ independent of $j$. By taking $j \rightarrow \infty$ we get the function $g_{i}$, which obviously satisfies the first two identities in the conclusion of Lemma 2.4. By the fact that $g_{i}^{j}(x)$ minimizes the Dirichlet integral among all Lipschitz functions with the same boundary data we know that $\int_{M}\left|\nabla g_{i}^{j}\right|^{2} d v$ is a decreasing sequence of $j$. This establishes the third property of $g_{i}$.

Using the barrier functions on each end we can construct linearly independent bounded harmonic functions as in [L-T2]. 
Lemma 2.5. Let $M^{m}$ be a minimal submanifold in $R^{n+1}, K \subset M$ be a compact subset in $M, E_{i}$ be the ends with respect to $K$, then there exist linearly independent harmonic functions $u_{i}$ on $M$ which satisfy that

$$
\lim _{x \rightarrow \infty_{x \in E_{i}}} u_{i}(x)=1, \lim _{x \rightarrow \infty} u_{x \in E_{i}} u_{i}(x)=0, \int_{E_{i}}\left|\nabla u_{i}\right|^{2} d v<\infty .
$$

Proof. For the completeness we sketch the proof here too. Let $u_{i}^{j}$ to be the harmonic function on $B_{p}\left(r_{j}\right)$ satisfying that

$$
\left.u_{i}^{j}(x)\right|_{\partial B_{p}\left(r_{j}\right) \cap E_{k}}=0, \text { for } k \neq i,\left.u_{i}^{j}(x)\right|_{\partial B_{p}\left(r_{j}\right) \cap E_{i}}=1 .
$$

Using the barrier functions we construct in Lemma 2.4 and taking $j \rightarrow \infty$ we have the bounded harmonic functions. For the proof of finite Dirichlet integral one can apply Lemma 1.4 of [L-T2].

Proposition 2.6. Let $M^{m}$ be a minimal submanifold in $R^{n+1}$. If $M^{m}$ has Euclidean volume growth, i.e.,

$$
\lim _{r \rightarrow \infty} \frac{\operatorname{Vol}\left(D_{p}(r)\right)}{r^{m}}<\infty
$$

then $M^{m}$ has finite many ends.

Proof. By Lemma 2.5 we know that the number of ends is controlled by the dimension of bounded harmonic functions with finite Dirichlet integral. In the case when $M$ has Euclidean volume growth, combining with the fact that $\frac{\operatorname{Vol}\left(D_{p}(r)\right)}{r^{m}}$ (cf. [Ty]) is a increasing function of $r$, we know that $M$ has the volume doubling property. Since, by Corollary 2.2, the mean value property holds for the subharmonic functions, applying a general theorem of Peter $\mathrm{Li}$ (cf. Theorem 1 in [L2], also [C-M]) we know that the polynomial growth harmonic function space is of finite dimension. In particular, the dimension of bounded harmonic function space is finite. Therefore $M$ has only finite many ends.

Remark. By a result of M. Anderson (cf. [An]), we know that if $M$ has finite total scalar curvature $\int_{M}\|A\|^{n} d v$ then $M$ has at most Euclidean volume growth. Therefore we have the following corollary, which can also be proved using the scaling argument and the Gromov compactness theorem (cf. [G-L-P]): 
Corollary 2.7. Let $M^{m}$ be minimal submanifold in $R^{n+1}$ with finite total scalar curvature. Then $M$ has finite many ends.

Similarly, we can have the following results on the finiteness of the number of ends.

Proposition 2.8. Let $M^{m}$ be minimal submanifold of dimension $m$ in $R^{n+1}$ and let $\|A\|^{2}(x)$ be the square of the length of the second fundamental form. If we have $\|A\|^{2}(x) \leq k(\rho(x))$, where $k(t)$ is a nonincreasing continuous function such that $\int_{0}^{\infty} \rho^{n-1} k(\rho) d \rho<\infty$, then $M$ has only finite ends.

Proof. As in the previous proposition it suffices to prove that the bounded harmonic function space is of finite dimension. Since in the case $M$ is a minimal submanifold in $R^{n+1}$ we have $\operatorname{Ricci}_{M}(x) \geq-\|A\|^{2}(x) \geq-k(\rho(x))$, a theorem of Li-Tam (See [L-T2]) says that under the assumption of our proposition the bounded harmonic function space is of finite dimension. Therefore $M$ has only finite many ends.

At the end we write the following result for the case when $M$ is a Kähler manifold.

Proposition 2.9. Let $M^{m}(m \geq 4)$, a Kähler manifold of real dimension $m$, be a complete minimal submanifold in $R^{n+1}$, and let $\|A\|^{2}(x)$ be the square of the length of the second fundamental form. If $\|A\|$ is of square integrable then $M$ has only one end.

Proof. Just as what we did before we only need to show that there is no nonconstant harmonic function $u$ on $M$ such that $u$ has finite Dirichlet integral.

First since $u$ has finite Dirichlet integral, by Lemma 3.1 of [L1] (see also $[\mathrm{Gr}]$ ) we know that $u$ is in fact a pluriharmonic function. Let $v=|\nabla u|^{2}$. Once we know $u$ is a pluriharmonic function we can sharpen the Bochner formula for $v=|\nabla u|$ to get the following inequality:

$$
\Delta v-\frac{|\nabla v|^{2}}{v} \geq-\|A\|^{2}(x) v .
$$

The interested reader can consult Lemma 3.2 of [L1] for a proof of the above inequality. Since we also know that there exists positive Green's function 
on $M$ by Lemma 2.1, we can apply Li-Yau's theorem (cf. Corollary 2.2 of [L-Y1]) to conclude $v$ is zero, i.e., $u$ is a constant.

Remark. Note that the situation here is totally different from the complex dimension one case. When $M$ is a Riemann surface one can find examples of minimal surfaces with finite total curvature and many ends. On the other hand a known result says that if $M$ is a complete minimal immersed surfaces in $R^{3}$ with finite total curvature and only one embedded end then $M$ is a plane.

\section{Gap theorems.}

First we can show the following gap theorem on the number of ends of minimal submanifolds in $R^{n+1}$.

Theorem 3.1. Let $M^{m}(m \geq 3)$ be a complete minimal immersed submanifold in $R^{n+1}$, and let $S(m)$ be the Sobolev constant in Corollary 2.3. If

$$
\left(\int_{M}\|A\|^{m} d v\right)^{\frac{1}{m}}<C_{2}(m)=\sqrt{\frac{m}{m-1} S^{-1}}
$$

then $M$ has only one end.

Proof. We argue by contradiction. By the construction of last section we learn that if $M$ has more than one end then there exists a nontrivial bounded harmonic function $u(x)$ on $M$ which has finite total energy. Let $f(x)=|\nabla u|$. The Bochner formula from $[\mathrm{S}-\mathrm{Y}]$ yields

$$
f \Delta f+\|A\|^{2}(x) f^{2} \geq \frac{1}{m-1}|\nabla f|^{2} .
$$

Let $\varphi$ be a cut-off function such that

$$
\varphi(x)= \begin{cases}1 & \text { if } x \in B_{p}(r) \\ 0 & \text { if } x \in M \backslash B_{p}(2 r),\end{cases}
$$

and

$$
|\nabla \varphi| \leq \frac{C}{r}, \text { with } C=2,
$$


Multiplying $\varphi^{2}$ on both sides of the above inequality (3.1) and integrating by parts we can write

$$
\begin{aligned}
& -\int_{M}|\nabla f|^{2} \varphi^{2} d v-2 \int_{M}\langle\nabla f, \nabla \varphi\rangle f \varphi d v+\int_{M}\|A\|^{2} f^{2} \varphi^{2} d v \\
& \geq \frac{1}{m-1} \int_{M}|\nabla f|^{2} \varphi^{2} d v .
\end{aligned}
$$

Using Schwartz inequality, for any positive number $I>0$, we have

$$
\int_{M}\|A\|^{2} f^{2} \varphi^{2} d v+\frac{1}{I} \int_{M} f^{2}|\nabla \varphi|^{2} d v \geq\left(\frac{m}{m-1}-I\right) \int_{M}|\nabla f|^{2} \varphi^{2} d v
$$

On the other hand, the Sobolev inequality yields

$$
\int_{M}|\nabla(f \varphi)|^{2} d v \geq S^{-1}\left(\int_{M}(f \varphi)^{\frac{2 m}{m-2}} d v\right)^{\frac{m-2}{m}} .
$$

Simple calculation together with Schwartz inequality yields

$$
\begin{aligned}
& (I I+1) \int_{M}|\nabla f|^{2} \varphi^{2} d v \\
& \geq S^{-1}\left(\int_{M}(f \varphi)^{\frac{2 m}{m-2}} d v\right)^{\frac{m-2}{m}}-\left(1+\frac{1}{I I}\right) \int_{M} f^{2}|\nabla \varphi|^{2} d v,
\end{aligned}
$$

where $I I$ is a positive real number which will be chosen later. Combining (3.2) and (3.3) we have

$$
\begin{aligned}
\int_{M}\|A\|^{2} f^{2} \varphi^{2} d v \geq & \frac{\left(\frac{m}{m-1}-I\right) S^{-1}}{I I+1}\left(\int_{M}(f \varphi)^{\frac{2 m}{m-2}} d v\right)^{\frac{m-2}{m}} \\
& -\left(\frac{1}{I}+\frac{\frac{m}{m-1}-I}{I I}\right) \int f^{2}|\nabla \varphi|^{2} d v
\end{aligned}
$$

Now applying Hörder inequality to the left hand side of the above inequality we can have

$$
\begin{aligned}
& \left(\int_{M}\|A\|^{m} d v\right)^{\frac{1}{m}}\left(\int_{M}(f \varphi)^{\frac{2 m}{m-2}} d v\right)^{\frac{m-2}{m}} \\
& \geq \frac{\left(\frac{m}{m-1}-I\right) S^{-1}}{I I+1}\left(\int_{M}(f \varphi)^{\frac{2 m}{m-2}} d v\right)^{\frac{m-2}{m}} \\
& -\left(\frac{1}{I}+\frac{\frac{m}{m-1}-I}{I I}\right) \int_{M} f^{2}|\nabla \varphi|^{2} d v
\end{aligned}
$$


Finally we have

$$
\begin{aligned}
& \left(\frac{1}{I}+\frac{\frac{m}{m-1}-I}{I I}\right) \int_{M} f^{2}|\nabla \varphi|^{2} d v \\
& \geq\left(\frac{\left(\frac{m}{m-1}-I\right) S^{-1}}{I I+1}-\left(\int_{M}\|A\|^{m} d v\right)^{\frac{1}{m}}\right)\left(\int_{M}(f \varphi)^{\frac{2 m}{m-2}} d v\right)^{\frac{m-2}{m}} .
\end{aligned}
$$

Choosing $I$ and $I I$ small enough one can make

$$
\left(\frac{\left(\frac{m}{m-1}-I\right) S^{-1}}{I I+1}-\left(\int_{M}\|A\|^{m} d v\right)^{\frac{1}{m}}\right) \geq \epsilon>0 .
$$

Then we have

$$
\left(\frac{1}{I}+\frac{\frac{m}{m-1}-I}{I I}\right) \int_{M} f^{2}|\nabla \varphi|^{2} d v \geq \epsilon\left(\int_{M}(f \varphi)^{\frac{2 m}{m-2}} d v\right)^{\frac{m-2}{m}}
$$

Letting $r \rightarrow \infty$ we will have

$$
\int_{M} f^{\frac{2 m}{m-2}} d v \leq 0
$$

which implies that $f \equiv 0$ and therefore $u$ is a constant function. The contradiction here shows that $M$ has at most one end.

Remarks. The first remark we want to make is that the similar argument can give an improvement of a lower bound estimate of Li-Yau (cf. [L-Y2]) on the eigenvalue of the Schrödinger operator. More precisely we can have the following results.

Let $q(x)$ be a positive function defined on $D$, a domain in $R^{n}$. We consider the operator

$$
\frac{\Delta}{q(x)}
$$

The lower bound estimate for the first eigenvalue of the above operator proved by Li-Yau concludes that.

$$
\mu_{1} \geq \frac{n(n-2)}{4 e}\left(\Omega_{n-1}\right)^{\frac{2}{n}}\|q\|_{L^{\frac{n}{2}}}^{-1}
$$

where $\Omega_{n-1}$ is the area of the unit $(n-1)$-sphere, $e$ is the Euler number. The same argument as in the proof of Theorem 3.1 gives a slight improvement on the above estimate. A direct trace of the calculation can show

$$
\mu_{1} \geq \frac{n(n-2)}{4}\left(\Omega_{n-1}\right)^{\frac{2}{n}}\|q\|_{L^{\frac{n}{2}}}^{-1}
$$


The second remark is that we do not know whether the assumption $m>2$ is necessary or not. There is a theorem by Fischer-Colbrie [F-C] saying that if $M$ is a minimal surface in $R^{3}$ with finite total curvature then $M$ has finite Morse index. By Huber's theorem we also know that $M$ has finite many ends. In [C-S-Z], using the Liouville type theorem of SchoenYau for the stable minimal hypersurfaces they showed that if $M$ is a stable hypersurface in $R^{n+1}$ then $M$ has only one end. Comparing to their result we neither assume that $M$ is a stable nor $M$ is a hypersurface.

Now we begin the proof of Theorem A. Namely we will show that if the total scalar curvature is smaller than a constant $C_{1}(n)$ then the minimal hypersurface $M$ has to be a hyperplane. We believe that the similar result also holds for minimal submanifolds.

Theorem 3.2. Let $M^{n}(n \geq 4)$ be a complete minimal immersed hypersurface in $R^{n+1}$, and let $S(n)$ be the Sobolev constant in Corollary 2.3. If

$$
\left(\int_{M}\|A\|^{n} d v\right)^{\frac{1}{n}}<C_{1}(n)=\sqrt{\frac{4(n-1)}{(n-2) n} S^{-1}}
$$

then $M$ has to be a hyperplane.

Before we prove Theorem 3.2, we need the following Lemma.

Lemma 3.3. Let $M^{n}(n \geq 4)$ be a complete minimal immersed hypersurface in $R^{n+1}$. If the total scalar curvature $\int_{M}\|A\|^{n} d v$ is finite then $\int_{M}\|A\|^{n-2} d v$ is also finite.

Proof. First, by Theorem 3.3 of [S-Z] we know that the rescaling sequences $M_{i}=\left\{\frac{1}{r_{i}} M\right\}$ converging smoothly to a flat open Riemannian manifold $M_{\infty}$ in the sense of Cheeger-Gromov, to which we can attach one point $O$ such that $\overline{M_{\infty}}=M_{\infty} \cup O$ is the union of several hyperplanes through the origin $O$ and $O$ is the only singularity of $\overline{M_{\infty}}$. At the mean time, by Corollary 2.7 we know that $M$ has only finite many ends. Therefore we only need to show that $\int_{E}\|A\|^{n-2} d v$ is finite for each end $E$. Because of the convergence of the rescaling sequences $\left\{M_{i}\right\}$ we know that the end $E$, with respect to big enough compact subset is a graph over the tangent space at infinity. Now we can use an estimate of Schoen (Proposition 3 of [Sc]) to get the following uniform estimate of $\|A\|(x)$ :

$$
\|A\|(x) \leq \frac{C}{r^{n}}
$$


for $x \in M \cap \partial D_{p}(r)$ and $r \gg 1$.

On the other hand, by Theorem 4.1 of [An], we have

$$
\operatorname{Vol}\left(D_{p}(k+1) \backslash D_{p}(k)\right) \leq C^{\prime}(k+1)^{n},
$$

for some uniform constant $C^{\prime}$. Therefore we have

$$
\begin{aligned}
\int_{M}\|A\|^{n-2} d v & =\sum_{k=0}^{\infty} \int_{M \cap\left(D_{p}(k+1) \backslash D_{p}(k)\right)}\|A\|^{n-2} d v \\
& \leq C_{3}+C_{4}\left(\sum_{k=0}^{\infty} \frac{(k+1)^{n}}{k^{n(n-2)}}\right) \\
& <\infty .
\end{aligned}
$$

Now we can prove Theorem 3.2.

Proof of Theorem 3.2. We first need the following sharp version of Simon's inequality due to Simon, Schoen and Yau on the length of the second fundamental form.

$$
\|A\| \Delta\|A\|+\|A\|^{4} \geq \frac{2}{n}|\nabla\|A\||^{2} .
$$

One can consult [S-S-Y] for the proof of a more general formula.

Let $\varphi$ be a cut-off function as in the proof of Theorem 3.1. Multiplying $\|A\|^{n-4} \varphi^{2}$ on both sides of the above inequality and integrating by parts we have that

$$
\begin{aligned}
& -(n-3) \int_{M}|\nabla\|A\||^{2}\|A\|^{n-4} \varphi^{2} d v-2 \int_{M}\langle\nabla\|A\|, \nabla \varphi\rangle\|A\|^{n-3} \varphi d v \\
& +\int_{M}\|A\|^{n} \varphi^{2} d v \geq \frac{2}{n} \int_{M}|\nabla\|A\||^{2}\|A\|^{n-4} \varphi^{2} d v .
\end{aligned}
$$

Applying Schwartz inequality, we can write

$$
\begin{aligned}
& \left((n-3)+\frac{2}{n}-I I\right) \int_{M} \mid \nabla\|A\|^{2}\|A\|^{n-4} \varphi^{2} d v \\
& \leq \frac{1}{I I} \int_{M}\|A\|^{n-2}|\nabla \varphi|^{2} d v+\int_{M}\|A\|^{n} \varphi^{2} d v
\end{aligned}
$$

for any positive number $I I$. 
On the other hand, direct calculation yields the following inequality, after using the Schwartz inequality.

$$
\begin{aligned}
\int_{M}\left|\nabla\left(\|A\|^{\frac{n-2}{2}} \varphi\right)\right|^{2} d v \leq & (1+I)\left(\frac{n-2}{2}\right)^{2} \int_{M}|\nabla\|A\||^{2}\|A\|^{n-4} \varphi^{2} d v \\
& +\left(1+\frac{1}{I}\right) \int_{M}\|A\|^{n-2}|\nabla \varphi|^{2} d v
\end{aligned}
$$

for any positive number $I$.

Combining (3.5) and and above inequality together we have

$$
\begin{aligned}
\int_{M}\left|\nabla\left(\|A\|^{\frac{n-2}{2}} \varphi\right)\right|^{2} d v \leq & \frac{(1+I)\left(\frac{n-2}{2}\right)^{2}}{n-3+\frac{2}{n}-I I} \int_{M}\|A\|^{n} \varphi^{2} d v \\
& +\frac{(1+I)\left(\frac{n-2}{2}\right)^{2}}{\left(n-2+\frac{2}{n}-I I\right) I I} \int_{M}\|A\|^{n-2}|\nabla \varphi|^{2} d v \\
& +\left(1+\frac{1}{I}\right) \int_{M}\|A\|^{n-2}|\nabla \varphi|^{2} d v
\end{aligned}
$$

Applying Sobolev inequality one can write

$$
\begin{aligned}
& S^{-1}\left(\int\|A\|^{\frac{n-2}{2} \frac{2 n}{n-2}} \varphi^{\frac{2 n}{n-2}} d v\right)^{\frac{n-2}{n}} \\
& \leq \frac{(1+I)\left(\frac{n-2}{2}\right)^{2}}{n-3+\frac{2}{n}-I I} \int_{M}\|A\|^{n} \varphi^{2} d v \\
& \quad+\frac{(1+I)\left(\frac{n-2}{2}\right)^{2}}{\left(n-3+\frac{2}{n}-I I\right) I I} \int_{M}\|A\|^{n-2}|\nabla \varphi|^{2} d v \\
& \quad+\left(1+\frac{1}{I}\right) \int_{M}\|A\|^{n-2}|\nabla \varphi|^{2} d v .
\end{aligned}
$$

Now let $r \rightarrow \infty$. Use Lemma 3.3 we have

$$
\left(S^{-1}-\frac{(1+I)\left(\frac{n-2}{2}\right)^{2}}{n-3+\frac{2}{n}-I I}\left(\left(\int_{M}\|A\|^{n} d v\right)^{\frac{1}{n}}\right)^{2}\right)\left(\int_{M}\|A\|^{n} d v\right)^{\frac{n-2}{n}} \leq 0 .
$$

Choosing $I$ and $I I$ small enough one can easily find a positive number $\epsilon$ such that

$$
S^{-1}-\frac{(1+I)\left(\frac{n-2}{2}\right)^{2}}{n-3+\frac{2}{n}-I I}\left(\left(\int_{M}\|A\|^{n} d v\right)^{\frac{1}{n}}\right)^{2} \geq \epsilon>0
$$


Then we can conclude that $\|A\| \equiv 0$. Therefore, $M$ is a totally geodesic hyperplane.

Added in proof: It turns out that, for $n \geq 6$, the constant in Theorem A can be improved by using a trick in [L-Y2] and Theorem 4.2 of [S-Z].

Theorem $\mathbf{A}^{\prime}$. With the notations in Theorem $A$, if $\|A\|_{L^{n}} \leq \sqrt{S^{-1}(n)}$, then $M$ is a hyperplane.

Proof. From the remark after the proof of Theorem 3.1 (just carry the similar calculation for general manifold) we know that,

$$
\mu_{1} \geq S^{-1}(n)\|q\|_{L^{\frac{n}{2}}}^{-1}
$$

where $\mu_{1}$ is the first eigenvalue of the operator $\frac{\Delta}{q}$ and $S(n)$ is the $L^{2}$-Sobolev constant as defined in (2.3). On the other hand, it is easy to see that if $(\Delta+q) \varphi=-\lambda \varphi$ has an eigenvalue $\lambda<0$ then $\Delta \phi=-\mu q(x) \phi$ has an eingenvalue $\mu<1$ (cf. [L-Y2]). Therefore, if $\|A\|_{L^{n}} \leq \sqrt{S(n)^{-1}}$ we have

$$
\mu_{1}\left(-\frac{\Delta}{\|A\|^{2}}\right) \geq 1
$$

Hence

$$
\lambda_{1}\left(-\left(\Delta+\|A\|^{2}\right)\right) \geq 0 .
$$

Namely $M$ is a stable hypersurface. Combining with Theorem 4.2 of [S-Z] we conclude that $M$ is a hyperplane.

\section{References.}

[Al] W.K. Allard, First variation of a varifold, Annals of Math., 95 (1972), 417-491.

[An] M. Anderson, The compactification of a minimal submanifold in Euclidean space by the Guass map, preprint (final version in Dept. of Math, California Institute of Technology, Pasadena, CA 91125), 1986.

[C-D-K] S.S. Chern, M. Do Carmo and S. Kobayashi, Minimal submanifolds of a sphere with second fundamental form of constant length. Functional Analysis and Related Fields, Springer-Verlag, (1970), $59-75$. 
[C-L-Y] S-Y. Cheng, P. Li and S.-T. Yau, Heat equations on minimal submanifolds and their applications, Amer. J. Math., 103 (1981), 1021-1063.

[C-M] T. Colding and W. Minicozzi, Weyl type bounds for harmonic functions, Invent. Math., 131 (1998), 257-298.

[C-S-Z] H. Cao, Y. Shen and S. Zhu, The structure of stable minimal hypersurfaces in $R^{n+1}$, Math. Res. Letter, 4 (1997), 637-645.

[F-C] D. Fischer-Colbrie, On the complete minimal surfaces with finite Morse index in three manifolds, Invent. Math., 82 (1985), 121132.

[Gr] M. Gromov, Kähler hyperbolicity and $L_{2}$-Hodge theory, J. Diff. Geom., 33 (1991), 263-292.

[G-L-P] M. Gromov, J. Lafontaine, and P. Pansu, Structure metriqué pour les varieites Riemanniennes, Nathen, Cedic/Fernand, 1981.

[L1] P. Li, On the structure of complete Kähler manifolds with nonnegative curvature near infinity, Invent. Math., 99 (1990), 579-600.

[L2] P. Li, On polynomial growth harmonic sections, Math. Res. Letter, 4 (1997), 35-44.

[L-T1] P. Li and L.F. Tam, Symmetric Green's functions on complete manifolds, Amer. J. Math., 109 (1987), 1129-1154.

[L-T2] P. Li and L.F. Tam, Harmonic functions and the structure of complete manifolds, J. Diff. Geom., 35 (1992), 359-383.

[L-W] P. Li and J. Wang, Mean value inequalities, preprint.

[L-Y1] P. Li and S.-T. Yau, Curvature and holomorphic mappings of complete Kähler manifolds, Compositio. Mathematica, 73 (1990), 125144.

[L-Y2] P. Li and S.-T. Yau, On the Schrödinger equation and the eigenvalue problems, Comm. Math. Phys., 88 (1983), 309-318.

[M-S] J. Michael and L.M. Simon, Sobolev and mean-vaule inequalities on generalized submanifolds of $R^{n}$, Comm. Pure Appl. Math., 26 (1973), 361-379. 
[Sc] R. Schoen, Uniqueness, symmetry, and embeddedness of minimal surfaces, J. Diff. Geom., 18 (1983), 791-809.

[S-Y] R. Schoen and S.-T. Yau, Harmonic maps and the topology of stable hypersurfaces and manifolds with non-negative Ricci curvature, Comment. Math. Helvetici, 51 (1976), 333-341.

[S-Z] Y. Shen and X. Zhu, On the stable complete minimal hypersurfaces in $\mathbf{R}^{n+1}$, Amer. J. Math., 120 (1998), 103-116.

[S-S-Y] R. Schoen, L. Simon and S.-T. Yau, Curvature estimate for minimal hypersurfaces, Acta. Math., 134 (1974), 275-288.

[S] J. Simons, Minimal varieties in Riemannian manifolds, Annals. of Math., 88 (1968), 62-105.

[Ty] T. Tysk, Finiteness of index and total scalar curvature for minimal hypersurfaces, Proc. Amer. Math. Soc., 105 (1989), 429-435.

[V] N. Varopoulos, Hardy-Littlewood theory for semigroups, J. Funct. Anal., 63 (1985), 240-260.

Department of Mathematics

STANFORD UNIVERSITY

STANFORD, CA 94305

E-mail address: Ini@math.stanford.edu

ReCeived June 16, 1999. 